\title{
Generalized $\alpha-\psi$-contractive type mappings of integral type and related fixed point theorems
}

\author{
Erdal Karapınar ${ }^{1,2}$, Priya Shahi $^{3}$ and Kenan Tas ${ }^{4 *}$
}

\section{"Correspondence:}

kenan@cankaya.edu.tr

${ }^{4}$ Department Mathematics and

Computer Science, Cankaya

University, Ankara, Turkey

Full list of author information is

available at the end of the article

\begin{abstract}
The aim of this paper is to introduce two classes of generalized $\alpha$ - $\psi$-contractive type mappings of integral type and to analyze the existence of fixed points for these mappings in complete metric spaces. Our results are improved versions of a multitude of relevant fixed point theorems of the existing literature.

MSC: $54 \mathrm{H} 25 ; 47 \mathrm{H} 10 ; 54 \mathrm{E} 50$
\end{abstract}

Keywords: fixed point; complete metric space; contractive mapping; partial order

\section{Introduction and preliminaries}

Recently, Samet et al. [1] introduced a very interesting notion of $\alpha-\psi$-contractions via $\alpha$-admissible mappings. In this paper, the authors [1] proved the existence and uniqueness of a fixed point for such a class of mappings in the context of complete metric spaces. Furthermore, the famous Banach [2] fixed point result was observed as a consequence of their main results. Following this initial paper, several authors have published new fixed point results by modifying, improving and generalizing the notion of $\alpha-\psi$-contractions in various abstract spaces; see, e.g., [3-8]. Very recently, Shahi et al. [9] gave the integral version of $\alpha-\psi$-contractive type mappings and proved some related fixed point theorems. As a consequence of the main results of this paper [9], the well-known integral contraction theorem of Branciari [10] and hence the celebrated Banach contraction principle were obtained.

In the present work, we introduce two classes of generalized $\alpha$ - $\psi$-contractive type mappings of integral type inspired by the report of Karapınar and Samet [7]. Also, we analyze the existence and uniqueness of fixed points for such mappings in complete metric spaces. Our results generalize, improve and extend not only the results derived by Shahi et al. [9], Samet et al. [1] and Branciari [10] but also various other related results in the literature. Moreover, from our fixed point theorems, we will derive several fixed point results on metric spaces endowed with a partial order.

We recall some necessary definitions and basic results from the literature. Throughout the paper, let $\mathbb{N}$ denote the set of all nonnegative integers.

Berzig and Rus [4] introduced the following definition. 
Definition 1.1 (see [4]) Let $N \in \mathbb{N}$. We say that $\alpha$ is $N$-transitive (on $X$ ) if

$$
x_{0}, x_{1}, \ldots, x_{N+1} \in X: \quad \alpha\left(x_{i}, x_{i+1}\right) \geq 1
$$

for all $i \in\{0,1, \ldots, N\} \Rightarrow \alpha\left(x_{0}, x_{N+1}\right) \geq 1$.

In particular, we say that $\alpha$ is transitive if it is 1 -transitive, i.e.,

$$
x, y, z \in X: \quad \alpha(x, y) \geq 1 \quad \text { and } \quad \alpha(y, z) \geq 1 \quad \Rightarrow \quad \alpha(x, z) \geq 1 .
$$

As consequences of Definition 1.1, we obtain the following remarks.

\section{Remark 1.1 (see [4])}

(1) Any function $\alpha: X \times X \rightarrow[0,+\infty)$ is 0 -transitive.

(2) If $\alpha$ is $N$ transitive, then it is $k N$-transitive for all $k \in \mathbb{N}$.

(3) If $\alpha$ is transitive, then it is $N$-transitive for all $N \in \mathbb{N}$.

(4) If $\alpha$ is $N$-transitive, then it is not necessarily transitive for all $N \in \mathbb{N}$.

Let $\Psi$ be a family of functions $\psi:[0, \infty) \rightarrow[0, \infty)$ satisfying the following conditions:

(1) $\psi$ is nondecreasing.

(2) $\sum_{n=1}^{+\infty} \psi^{n}(t)<\infty$ for all $t>0$, where $\psi^{n}$ is the $n$th iterate of $\psi$.

In the literature, such mappings are called in two different ways: (c)-comparison functions in some sources (see, e.g., [11]), and Bianchini-Grandolfi gauge functions in some others (see, e.g., [12-14]).

It can be easily verified that if $\psi$ is a (c)-comparison function, then $\psi(t)<t$ for any $t>0$. Define $\Phi=\left\{\varphi: \varphi: \mathbb{R}^{+} \rightarrow \mathbb{R}\right\}$ such that $\varphi$ is nonnegative, Lebesgue integrable and satisfies

$$
\int_{0}^{\epsilon} \varphi(t) d t>0 \quad \text { for each } \epsilon>0
$$

Shahi et al. in [9] introduced the following new concept of $\alpha-\psi$-contractive type mappings of integral type.

Definition 1.2 Let $(X, d)$ be a metric space and $T: X \rightarrow X$ be a given mapping. We say that $T$ is an $\alpha-\psi$-contractive mapping of integral type if there exist two functions $\alpha: X \times X \rightarrow$ $[0,+\infty)$ and $\psi \in \Psi$ such that for each $x, y \in X$,

$$
\alpha(x, y) \int_{0}^{d(T x, T y)} \varphi(t) d t \leq \psi\left(\int_{0}^{d(x, y)} \varphi(t) d t\right)
$$

where $\varphi \in \Phi$.

In what follows, we recollect the main results of Shahi et al. [9].

Theorem 1.1 [9] Let $(X, d)$ be a complete metric space and $\alpha: X \times X \rightarrow[0,+\infty)$ be a transitive mapping. Suppose that $T: X \rightarrow X$ is an $\alpha-\psi$-contractive mapping of integral type and satisfies the following conditions:

(i) $T$ is $\alpha$-admissible;

(ii) there exists $x_{0} \in X$ such that $\alpha\left(x_{0}, T x_{0}\right) \geq 1$; 
(iii) $T$ is continuous.

Then $T$ has a fixed point, that is, there exists $z \in X$ such that $T z=z$.

Theorem 1.2 [9] Let $(X, d)$ be a complete metric space and $\alpha: X \times X \rightarrow[0,+\infty)$ be a

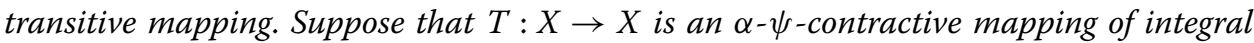
type and satisfies the following conditions:

(i) $T$ is $\alpha$-admissible;

(ii) there exists $x_{0} \in X$ such that $\alpha\left(x_{0}, T x_{0}\right) \geq 1$;

(iii) if $\left\{x_{n}\right\}$ is a sequence in $X$ such that $\alpha\left(x_{n}, x_{n+1}\right) \geq 1$ for all $n$ and $x_{n} \rightarrow x \in X$ as $n \rightarrow \infty$, then there exists a subsequence $\left\{x_{n(k)}\right\}$ of $\left\{x_{n}\right\}$ such that $\alpha\left(x_{n(k)}, x\right) \geq 1$ for all $k$.

Then $T$ has a fixed point, that is, there exists $z \in X$ such that $T z=z$.

Notice that in the theorems above, the authors proved only the existence of a fixed point. To guarantee the uniqueness of the fixed point, they needed the following condition.

(U): For all $x, y \in \operatorname{Fix}(T)$, there exists $z \in X$ such that $\alpha(x, z) \geq 1$ and $\alpha(y, z) \geq 1$, where $\operatorname{Fix}(T)$ denotes the set of fixed points of $T$.

\section{Main results}

In this section, we present our main results. First, we introduce two classes of generalized $\alpha-\psi$-contractive type mappings of integral type in the following way.

Definition 2.1 Let $(X, d)$ be a metric space and $T: X \rightarrow X$ be a given mapping. We say that $T$ is a generalized $\alpha-\psi$-contractive mapping of integral type I if there exist two functions $\alpha: X \times X \rightarrow[0,+\infty)$ and $\psi \in \Psi$ such that for each $x, y \in X$,

$$
\alpha(x, y) \int_{0}^{d(T x, T y)} \varphi(t) d t \leq \psi\left(\int_{0}^{M(x, y)} \varphi(t) d t\right)
$$

where $\varphi \in \Phi$ and $M(x, y)=\max \left\{d(x, y), d(x, T x), d(y, T y),\left[\frac{d(x, T y)+d(y, T x)}{2}\right]\right\}$.

Definition 2.2 Let $(X, d)$ be a metric space and $T: X \rightarrow X$ be a given mapping. We say that $T$ is a generalized $\alpha-\psi$-contractive mapping of integral type II if there exist two functions $\alpha: X \times X \rightarrow[0,+\infty)$ and $\psi \in \Psi$ such that for each $x, y \in X$,

$$
\alpha(x, y) \int_{0}^{d(T x, T y)} \varphi(t) d t \leq \psi\left(\int_{0}^{M(x, y)} \varphi(t) d t\right)
$$

where $\varphi \in \Phi$ and $M(x, y)=\max \left\{d(x, y),\left[\frac{d(x, T x)+d(y, T y)}{2}\right],\left[\frac{d(x, T y)+d(y, T x)}{2}\right]\right\}$.

Remark 2.1 It is evident that if $T: X \rightarrow X$ is an $\alpha$ - $\psi$-contractive mapping of integral type, then $T$ is a generalized $\alpha-\psi$-contractive mapping of integral types I and II.

The following is the first main result of this manuscript.

Theorem 2.1 Let $(X, d)$ be a complete metric space and $\alpha: X \times X \rightarrow[0,+\infty)$ be a transitive mapping. Suppose that $T: X \rightarrow X$ is a generalized $\alpha$ - $\psi$-contractive mapping of integral type I and satisfies the following conditions: 
(i) $T$ is $\alpha$-admissible;

(ii) there exists $x_{0} \in X$ such that $\alpha\left(x_{0}, T x_{0}\right) \geq 1$;

(iii) $T$ is continuous.

Then $T$ has a fixed point, that is, there exists $z \in X$ such that $T z=z$.

Proof Let $x_{0}$ be an arbitrary point of $X$ such that $\alpha\left(x_{0}, T x_{0}\right) \geq 1$. We construct an iterative sequence $\left\{x_{n}\right\}$ in $X$ in the following way:

$$
x_{n+1}=T x_{n} \quad \text { for all } n \geq 0 .
$$

If $x_{n_{0}}=x_{n_{0}+1}$ for some $n_{0}$, then, obviously, $x^{*}=x_{n_{0}}$ is a fixed point of $T$ and the proof is completed. Hence, from now on, we suppose that $x_{n} \neq x_{n+1}$ for all $n$. Due to the fact that $T$ is $\alpha$-admissible, we find that

$$
\alpha\left(x_{0}, x_{1}\right)=\alpha\left(x_{0}, T x_{0}\right) \geq 1 \Rightarrow \alpha\left(T x_{0}, T x_{1}\right)=\alpha\left(x_{1}, x_{2}\right) \geq 1
$$

Iteratively, we obtain that

$$
\alpha\left(x_{n}, x_{n+1}\right) \geq 1
$$

for all $n \geq 0$.

By applying inequality (3) with $x=x_{n-1}$ and $y=x_{n}$ and using (5), we deduce that

$$
\begin{aligned}
\int_{0}^{d\left(x_{n}, x_{n+1}\right)} \varphi(t) d t & =\int_{0}^{d\left(T x_{n-1}, T x_{n}\right)} \varphi(t) d t \leq \alpha\left(x_{n-1}, x_{n}\right) \int_{0}^{d\left(T x_{n-1}, T x_{n}\right)} \varphi(t) d t \\
& \leq \psi\left(\int_{0}^{M\left(x_{n-1}, x_{n}\right)} \varphi(t) d t\right),
\end{aligned}
$$

where

$$
\begin{aligned}
M\left(x_{n-1}, x_{n}\right) & =\max \left\{d\left(x_{n-1}, x_{n}\right), d\left(x_{n-1}, x_{n}\right), d\left(x_{n}, x_{n+1}\right), \frac{d\left(x_{n-1}, x_{n+1}\right)+d\left(x_{n}, x_{n}\right)}{2}\right\} \\
& \leq \max \left\{d\left(x_{n-1}, x_{n}\right), d\left(x_{n}, x_{n+1}\right)\right\} .
\end{aligned}
$$

By utilizing (7) and regarding the properties of the function $\psi$, we derive from (6) that

$$
\begin{aligned}
\int_{0}^{d\left(x_{n}, x_{n+1}\right)} \varphi(t) d t & =\int_{0}^{d\left(T x_{n-1}, T x_{n}\right)} \varphi(t) d t \leq \alpha\left(x_{n-1}, x_{n}\right) \int_{0}^{d\left(T x_{n-1}, T x_{n}\right)} \varphi(t) d t \\
& \leq \psi\left(\int_{0}^{\max \left\{d\left(x_{n-1}, x_{n}\right), d\left(x_{n}, x_{n+1}\right)\right\}} \varphi(t) d t\right) \\
& \leq \psi\left(\max \left\{\int_{0}^{d\left(x_{n-1}, x_{n}\right)} \varphi(t) d t, \int_{0}^{d\left(x_{n}, x_{n+1}\right)} \varphi(t) d t\right\}\right) \\
& \leq \psi\left(\int_{0}^{d\left(x_{n-1}, x_{n}\right)} \varphi(t) d t\right) .
\end{aligned}
$$

Notice that the case

$$
\int_{0}^{d\left(x_{n}, x_{n+1}\right)} \varphi(t) d t \leq \psi\left(\int_{0}^{d\left(x_{n}, x_{n+1}\right)} \varphi(t) d t\right)<\int_{0}^{d\left(x_{n}, x_{n+1}\right)} \varphi(t) d t
$$


is impossible due to the property $\psi(t)<t$ for all $t>0$. By using mathematical induction, we get, for all $n \in \mathbb{N}$,

$$
\int_{0}^{d\left(x_{n}, x_{n+1}\right)} \varphi(t) d t \leq \psi^{n}\left(\int_{0}^{d\left(x_{0}, x_{1}\right)} \varphi(t) d t\right)=\psi^{n}(d)
$$

where $d=\int_{0}^{d\left(x_{0}, x_{1}\right)} \varphi(t) d t$.

Letting $n \rightarrow+\infty$ in (9) and taking the property of $\psi$ on the account, we find that

$$
\int_{0}^{d\left(x_{n}, x_{n+1}\right)} \varphi(t) d t=0
$$

which, from (1), implies that

$$
d\left(x_{n}, x_{n+1}\right) \rightarrow 0 \quad \text { as } n \rightarrow \infty .
$$

We shall prove that $\left\{x_{n}\right\}$ is a Cauchy sequence. Suppose, on the contrary, that there exist an $\epsilon>0$ and subsequences $\{m(p)\}$ and $\{n(p)\}$ such that $m(p)<n(p)<m(p+1)$ with

$$
d\left(x_{m(p)}, x_{n(p)}\right) \geq \epsilon, \quad d\left(x_{m(p)}, x_{n(p)-1}\right)<\epsilon .
$$

Due to the definition of $M(x, y)$, we have that

$$
\begin{aligned}
M\left(x_{m(p)-1}, x_{n(p)-1}\right)= & \max \left\{d\left(x_{m(p)-1}, x_{n(p)-1}\right), d\left(x_{m(p)-1}, x_{m(p)}\right), d\left(x_{n(p)-1}, x_{n(p)}\right),\right. \\
& \left.\frac{d\left(x_{m(p)-1}, x_{n(p)}\right)+d\left(x_{n(p)-1}, x_{m(p)}\right)}{2}\right\} .
\end{aligned}
$$

By elementary evaluation, (11), we find that

$$
\lim _{p} \int_{0}^{d\left(x_{m(p)-1}, x_{m(p)}\right)} \varphi(t) d t=\lim _{p} \int_{0}^{d\left(x_{n(p)-1}, x_{n(p)}\right)} \varphi(t) d t=0
$$

In view of (11), (12) and the triangular inequality, we deduce that

$$
\begin{aligned}
d\left(x_{m(p)-1}, x_{n(p)-1}\right) & \leq d\left(x_{m(p)-1}, x_{m(p)}\right)+d\left(x_{m(p)}, x_{n(p)-1}\right) \\
& <\epsilon+d\left(x_{m(p)-1}, x_{m(p)}\right) .
\end{aligned}
$$

Letting $n \rightarrow \infty$ in the inequality above, we conclude that

$$
\lim _{p \rightarrow \infty} \int_{0}^{d\left(x_{m(p)-1}, x_{n(p)-1}\right)} \varphi(t) d t \leq \int_{0}^{\epsilon} \varphi(t) d t
$$

Owing to the transitivity of $\alpha$, we infer from (5) that

$$
\alpha\left(x_{m(p)-1}, x_{n(p)-1}\right) \geq 1 .
$$


Regarding inequality (3) and by using (16), we obtain

$$
\begin{aligned}
\int_{0}^{d\left(x_{\left.m(p), x_{n}(p)\right)}\right.} \varphi(t) d t & =\int_{0}^{d\left(T x_{m(p)-1}, T x_{n(p)-1}\right)} \varphi(t) d t \\
& \leq \alpha\left(x_{m(p)-1}, x_{n(p)-1}\right) \int_{0}^{d\left(T x_{m(p)-1}, T x_{n}(p)-1\right)} \varphi(t) d t \\
& \leq \psi\left(\int_{0}^{M\left(x_{m(p)-1}, x_{n(p)-1}\right)} \varphi(t) d t\right) .
\end{aligned}
$$

In view of (12) and using the triangular inequality, we get

$$
\begin{aligned}
t(m, n) & =\frac{d\left(x_{m(p)-1}, x_{n(p)}\right)+d\left(x_{n(p)-1}, x_{m(p)}\right)}{2} \\
& \leq \frac{d\left(x_{m(p)-1}, x_{m(p)}\right)+2 d\left(x_{m(p)}, x_{n(p)-1}\right)+d\left(x_{n(p)-1}, x_{n(p)}\right)}{2} \\
& <\frac{d\left(x_{m(p)-1}, x_{m(p)}\right)+d\left(x_{n(p)-1}, x_{n(p)}\right)}{2}+\epsilon .
\end{aligned}
$$

Therefore, using (11), we infer that

$$
\lim _{p} \int_{0}^{t(m, n)} \varphi(t) d t \leq \int_{0}^{\epsilon} \varphi(t) d t
$$

Now, from (3), (12), (13), (14), (15), (16) and (19), it then follows that

$$
\begin{aligned}
\int_{0}^{\epsilon} \varphi(t) d t & \leq \int_{0}^{d\left(x_{m(p)}, x_{n(p)}\right)} \varphi(t) d t \\
& \leq \alpha\left(x_{m(p)-1}, x_{n(p)-1}\right) \int_{0}^{d\left(T x_{m(p)-1}, T x_{n(p)-1}\right)} \varphi(t) d t \\
& \leq \psi\left(\int_{0}^{M\left(x_{m(p)-1}, x_{n(p)-1}\right)} \varphi(t) d t\right) \\
& \leq \psi\left(\int_{0}^{\epsilon} \varphi(t) d t\right),
\end{aligned}
$$

which is a contradiction. This implies that $\left\{x_{n}\right\}$ is a Cauchy sequence in $(X, d)$. Due to the completeness of $(X, d)$, there exists $z \in X$ such that $x_{n} \rightarrow z$ as $n \rightarrow+\infty$. The continuity of $T$ yields that $T x_{n} \rightarrow T z$ as $n \rightarrow+\infty$, that is, $x_{n+1} \rightarrow T z$ as $n \rightarrow+\infty$. By the uniqueness of the limit, we obtain $z=T z$. Therefore, $z$ is a fixed point of $T$.

Theorem 2.2 Let $(X, d)$ be a complete metric space and $\alpha: X \times X \rightarrow[0,+\infty)$ be a transitive mapping. Suppose that $T: X \rightarrow X$ is a generalized $\alpha-\psi$-contractive mapping of integral type I and satisfies the following conditions:

(i) $T$ is $\alpha$-admissible;

(ii) there exists $x_{0} \in X$ such that $\alpha\left(x_{0}, T x_{0}\right) \geq 1$;

(iii) if $\left\{x_{n}\right\}$ is a sequence in $X$ such that $\alpha\left(x_{n}, x_{n+1}\right) \geq 1$ for all $n$ and $x_{n} \rightarrow x \in X$ as $n \rightarrow \infty$, then there exists a subsequence $\left\{x_{n(k)}\right\}$ of $\left\{x_{n}\right\}$ such that $\alpha\left(x_{n(k)}, x\right) \geq 1$ for all $k$; 
(iv) $\psi$ is continuous for all $t>0$.

Then $T$ has a fixed point, that is, there exists $z \in X$ such that $T z=z$.

Proof From the proof of Theorem 2.1, we infer that the sequence $\left\{x_{n}\right\}$ defined by $x_{n+1}=T x_{n}$ for all $n \geq 0$ converges to $z \in X$. We obtain, from hypothesis (iii) and (3), that there exists a subsequence $\left\{x_{n(k)}\right\}$ of $x_{n}$ such that $\alpha\left(x_{n(k)}, z\right) \geq 1$ for all $k$. Now, applying inequality (3), we get, for all $k$,

$$
\begin{aligned}
\int_{0}^{d\left(x_{n(k)+1}, T z\right)} \varphi(t) d t & =\int_{0}^{d\left(T x_{n(k)}, T z\right)} \varphi(t) d t \leq \alpha\left(x_{n(k)}, z\right) \int_{0}^{d\left(T x_{n(k)}, T z\right)} \varphi(t) d t \\
& \leq \psi\left(\int_{0}^{M\left(x_{n(k)}, z\right)} \varphi(t) d t\right) .
\end{aligned}
$$

On the other hand, we have

$$
M\left(x_{n(k)}, z\right)=\max \left\{d\left(x_{n(k)}, z\right), d\left(x_{n(k)}, x_{n(k)+1}\right), d(z, T z), \frac{d\left(x_{n(k)}, T z\right)+d\left(z, x_{n(k)+1}\right)}{2}\right\} .
$$

Recall from the proof of Theorem 2.1 that the sequence $\left\{x_{n}\right\}$ converges to $z \in X$. Consequently, as $k \rightarrow \infty$, the limit of the terms $d\left(x_{n(k)}, z\right), d\left(x_{n(k)}, x_{n(k)+1}\right), d\left(z, x_{n(k)+1}\right)$ tends to 0 . Thus, by letting $k \rightarrow \infty$ in (22), we get that

$$
\lim _{k \rightarrow \infty} M\left(x_{n(k)}, z\right)=d(z, T z)
$$

Assume that $d(z, T z)>0$. In view of (23) and for $k$ large enough, we get $M\left(x_{n(k)}, z\right)>0$, which implies from (21) that

$$
\int_{0}^{d\left(x_{n(k)+1}, T z\right)} \varphi(t) d t \leq \psi\left(\int_{0}^{M\left(x_{n(k),}, z\right)} \varphi(t) d t\right)
$$

Letting $k \rightarrow \infty$ in (24) and by using (23), assumption (iv), together with the property of $\psi(t)<t$, we derive that

$$
\int_{0}^{d(z, T z)} \varphi(t) d t \leq \psi\left(\int_{0}^{d(z, T z)} \varphi(t) d t\right)<\int_{0}^{d(z, T z)} \varphi(t) d t
$$

which is a contradiction. Thus, we have $d(z, T z)=0$, that is, $z=T z$.

One can easily deduce the following result from Theorem 2.1.

Theorem 2.3 Let $(X, d)$ be a complete metric space and $\alpha: X \times X \rightarrow[0,+\infty)$ be a transitive mapping. Suppose that $T: X \rightarrow X$ is a generalized $\alpha-\psi$-contractive mapping of integral type II and satisfies the following conditions:

(i) $T$ is $\alpha$-admissible;

(ii) there exists $x_{0} \in X$ such that $\alpha\left(x_{0}, T x_{0}\right) \geq 1$;

(iii) $T$ is continuous.

Then $T$ has a fixed point, that is, there exists $z \in X$ such that $T z=z$.

In the next theorem, we exclude the continuity hypothesis of $T$ in Theorem 2.3 
Theorem 2.4 Let $(X, d)$ be a complete metric space and $\alpha: X \times X \rightarrow[0,+\infty)$ be a transitive mapping. Suppose that $T: X \rightarrow X$ is a generalized $\alpha$ - $\psi$-contractive mapping of integral type II and satisfies the following conditions:

(i) $T$ is $\alpha$-admissible;

(ii) there exists $x_{0} \in X$ such that $\alpha\left(x_{0}, T x_{0}\right) \geq 1$;

(iii) if $\left\{x_{n}\right\}$ is a sequence in $X$ such that $\alpha\left(x_{n}, x_{n+1}\right) \geq 1$ for all $n$ and $x_{n} \rightarrow x \in X$ as $n \rightarrow \infty$, then there exists a subsequence $\left\{x_{n(k)}\right\}$ of $\left\{x_{n}\right\}$ such that $\alpha\left(x_{n(k)}, x\right) \geq 1$ for all $k$.

Then $T$ has a fixed point, that is, there exists $z \in X$ such that $T z=z$.

Proof From the proof of Theorem 2.3, we infer that the sequence $\left\{x_{n}\right\}$ defined by $x_{n+1}=T x_{n}$ for all $n \geq 0$ converges to $z \in X$. We obtain, from hypothesis (iii) and (3), that there exists a subsequence $\left\{x_{n(k)}\right\}$ of $x_{n}$ such that $\alpha\left(x_{n(k)}, z\right) \geq 1$ for all $k$. Now, applying inequality (4), we get, for all $k$,

$$
\begin{aligned}
\int_{0}^{d\left(x_{n(k)+1}, T z\right)} \varphi(t) d t & =\int_{0}^{d\left(T x_{n(k)}, T z\right)} \varphi(t) d t \leq \alpha\left(x_{n(k)}, z\right) \int_{0}^{d\left(T x_{n(k)}, T z\right)} \varphi(t) d t \\
& \leq \psi\left(\int_{0}^{M\left(x_{n(k)}, z\right)} \varphi(t) d t\right) .
\end{aligned}
$$

On the other hand, we have

$$
\begin{aligned}
M\left(x_{n(k)}, z\right)= & \max \left\{d\left(x_{n(k)}, z\right), \frac{d\left(x_{n(k)}, x_{n(k)+1}\right)+d(z, T z)}{2},\right. \\
& \left.\frac{d\left(x_{n(k)}, T z\right)+d\left(z, x_{n(k)+1}\right)}{2}\right\} .
\end{aligned}
$$

Letting $k \rightarrow \infty$ in the above equality, we get that

$$
\lim _{k \rightarrow \infty} M\left(x_{n(k)}, z\right)=\frac{d(z, T z)}{2} .
$$

Assume that $d(z, T z)>0$. In view of (28) and for $k$ large enough, we get $M\left(x_{n}(k), z\right)>0$, which implies from (26) that

$$
\begin{aligned}
\int_{0}^{d\left(x_{n(k)+1}, T z\right)} \varphi(t) d t & \leq \psi\left(\int_{0}^{M\left(x_{n(k)}, z\right)} \varphi(t) d t\right) \\
& <\int_{0}^{M\left(x_{n(k)}, z\right)} \varphi(t) d t .
\end{aligned}
$$

Letting $k \rightarrow \infty$ in (29) and using (28), we obtain that

$$
\int_{0}^{d(z, T z)} \varphi(t) d t \leq \int_{0}^{\frac{d(z, T z)}{2}} \varphi(t) d t,
$$

which is a contradiction. Thus, we have $d(z, T z)=0$, that is, $z=T z$.

Remark 2.2 Notice that in Theorem 2.2, the continuity of $\psi$ is assumed as an extra condition. Despite Remark 2.1, Theorem 2.4 can be derived from Theorem 2.2 due to the additional assumption on $\psi$. 
In order to ensure the uniqueness of a fixed point of a generalized $\alpha$ - $\psi$-contractive mapping of integral type II, we need an additional condition (U) defined in the previous section.

Theorem 2.5 If the condition $(\mathrm{U})$ is added to the hypotheses of Theorem 2.1, then the fixed point $u$ of $T$ is unique.

Proof We shall show the uniqueness of a fixed point of $T$ by reductio ad absurdum. Suppose, on the contrary, that $v$ is another fixed point of $T$ with $v \neq u$. From the hypothesis (U), we obtain that there exists $z \in X$ such that

$$
\alpha(u, z) \geq 1, \quad \alpha(v, z) \geq 1 .
$$

Using the $\alpha$-admissible property of $T$, we get from (31) for all $n \in \mathbb{N}$

$$
\alpha\left(u, T^{n} z\right) \geq 1, \quad \alpha\left(v, T^{n} z\right) \geq 1
$$

Consider the sequence $\left\{z_{n}\right\}$ in $X$ by $z_{n+1}=T z_{n}$ for all $n \geq 0$ and $z_{0}=z$. From (32), for all $n$, we infer that

$$
\begin{aligned}
\int_{0}^{d\left(u, z_{n+1}\right)} \varphi(t) d t & =\int_{0}^{d\left(T u, T z_{n}\right)} \varphi(t) d t \leq \alpha\left(u, z_{n}\right) \int_{0}^{d\left(T u, T z_{n}\right)} \varphi(t) d t \\
& \leq \psi\left(\int_{0}^{M\left(u, z_{n}\right)} \varphi(t) d t\right) .
\end{aligned}
$$

On the other hand, we have

$$
\begin{aligned}
M\left(u, z_{n}\right) & =\max \left\{d\left(u, z_{n}\right), \frac{d(u, T u)+d\left(z_{n}, T z_{n}\right)}{2}, \frac{d\left(u, T z_{n}\right)+d\left(z_{n}, T u\right)}{2}\right\} \\
& =\max \left\{d\left(u, z_{n}\right), d(u, T u), d\left(z_{n}, T z_{n}\right), \frac{d\left(u, T z_{n}\right)+d\left(z_{n}, T u\right)}{2}\right\} \\
& =\max \left\{d\left(u, z_{n}\right), 0, d\left(z_{n}, z_{n+1}\right), \frac{d\left(u, z_{n+1}\right)+d\left(z_{n}, u\right)}{2}\right\} \\
& \leq \max \left\{d\left(u, z_{n}\right), d\left(z_{n}, z_{n+1}\right), \frac{d\left(u, z_{n+1}\right)+d\left(z_{n}, u\right)}{2}\right\} \\
& \leq \max \left\{d\left(u, z_{n}\right), d\left(z_{n}, z_{n+1}\right), d\left(u, z_{n+1}\right)\right\} .
\end{aligned}
$$

Due to the monotone property of $\psi$ and using the above inequality, we infer from (33) that

$$
\begin{aligned}
& \int_{0}^{d\left(u, z_{n+1}\right)} \varphi(t) d t \\
& \quad \leq \psi\left(\int_{0}^{M\left(u, z_{n}\right)} \varphi(t) d t\right) \\
& \quad \leq \psi\left(\int_{0}^{\max \left\{d\left(u, z_{n}\right), d\left(z_{n}, z_{n+1}\right), d\left(u, z_{n+1}\right)\right\}} \varphi(t) d t\right) \\
& \quad \leq \psi\left(\max \left\{\int_{0}^{d\left(u, z_{n}\right)} \varphi(t) d t, \int_{0}^{d\left(z_{n}, z_{n+1}\right)} \varphi(t) d t, \int_{0}^{d\left(u, z_{n+1}\right)} \varphi(t) d t\right\}\right)
\end{aligned}
$$


for all $n$. Let us examine the possibilities for the inequality above. For simplicity, let

$$
P\left(u, z_{n}\right)=\max \left\{\int_{0}^{d\left(u, z_{n}\right)} \varphi(t) d t, \int_{0}^{d\left(z_{n}, z_{n+1}\right)} \varphi(t) d t, \int_{0}^{d\left(u, z_{n+1}\right)} \varphi(t) d t\right\} .
$$

If $P\left(u, z_{n}\right)=\int_{0}^{d\left(u, z_{n+1}\right)} \varphi(t) d t$, then due to the properties of the function $\psi$, we get

$$
\int_{0}^{d\left(u, z_{n+1}\right)} \varphi(t) d t \leq \psi\left(\int_{0}^{d\left(u, z_{n+1}\right)} \varphi(t) d t\right)<\int_{0}^{d\left(u, z_{n+1}\right)} \varphi(t) d t
$$

which is a contradiction. If $P\left(u, z_{n}\right)=\int_{0}^{d\left(u, z_{n}\right)} \varphi(t) d t$, then

$$
\int_{0}^{d\left(u, z_{n+1}\right)} \varphi(t) d t \leq \psi\left(\int_{0}^{d\left(u, z_{n}\right)} \varphi(t) d t\right)
$$

thereby implying that

$$
\int_{0}^{d\left(u, z_{n+1}\right)} \varphi(t) d t \leq \psi^{n}\left(\int_{0}^{d\left(u, z_{0}\right)} \varphi(t) d t\right)
$$

for all $n \geq 1$. Letting $n \rightarrow \infty$ in the above inequality, we obtain that

$$
\lim _{n \rightarrow \infty} \int_{0}^{d\left(u, z_{n+1}\right)} \varphi(t) d t=0
$$

which from (1) implies that

$$
\lim _{n \rightarrow \infty} d\left(z_{n}, u\right)=0
$$

Let us analyze the last case: $P\left(u, z_{n}\right)=\int_{0}^{d\left(z_{n}, z_{n+1}\right)} \varphi(t) d t$. Regarding the properties of $\phi$ and the triangle inequality, we have

$$
d\left(z_{n}, z_{n+1}\right) \leq d\left(z_{n}, u\right)+d\left(u, z_{n+1}\right) \leq 2 \max \left\{d\left(z_{n}, u\right), d\left(u, z_{n+1}\right)\right\} .
$$

Notice that if $d\left(z_{n}, u\right) \leq d\left(u, z_{n+1}\right)$, then, as in the analysis of the first case, we get a contradiction. Hence,

$$
d\left(z_{n}, z_{n+1}\right) \leq d\left(z_{n}, u\right)+d\left(u, z_{n+1}\right) \leq 2 \max \left\{d\left(z_{n}, u\right), d\left(u, z_{n+1}\right)\right\} \leq 2 d\left(z_{n}, u\right)
$$

and hence we easily deduce that

$$
\begin{aligned}
\int_{0}^{d\left(u, z_{n+1}\right)} \varphi(t) d t & \leq \psi\left(\int_{0}^{d\left(z_{n}, z_{n+1}\right)} \varphi(t) d t\right) \\
& \leq \psi\left(\int_{0}^{d\left(z_{n}, u\right)+d\left(u, z_{n+1}\right)} \varphi(t) d t\right) \\
& \leq \psi\left(\int_{0}^{2 \max \left\{d\left(z_{n}, u\right), d\left(u, z_{n+1}\right)\right\}} \varphi(t) d t\right) \\
& \leq \psi\left(\int_{0}^{2 d\left(z_{n}, u\right)} \varphi(t) d t\right)
\end{aligned}
$$


for each $n$. Consequently, we find that

$$
\int_{0}^{d\left(u, z_{n+1}\right)} \varphi(t) d t \leq \psi^{n}\left(\int_{0}^{2 d\left(u, z_{0}\right)} \varphi(t) d t\right)
$$

for all $n \geq 1$. Letting $n \rightarrow \infty$ in the above inequality, we obtain that

$$
\lim _{n \rightarrow \infty} \int_{0}^{d\left(u, z_{n+1}\right)} \varphi(t) d t=0
$$

which from (1) implies that

$$
\lim _{n \rightarrow \infty} d\left(z_{n}, u\right)=0 .
$$

Similarly, we can show that

$$
\lim _{n \rightarrow \infty} d\left(z_{n}, v\right)=0
$$

From equations (41) and (42), we obtain that $u=v$. Therefore, we have proved that $u$ is the unique fixed point of $T$.

The following result can be easily deduced from Theorem 2.5 due to Remark 2.1.

Theorem 2.6 Adding the condition (U) to the hypotheses of Theorem 2.3 (resp. Theorem 2.4), one obtains that $u$ is the unique fixed point of $T$.

\section{Consequences}

In this section, we shall list some existing results in the literature that can be deduced easily from our Theorem 2.6.

\subsection{Standard fixed point theorems}

Theorem 1.1 and Theorem 1.2 are immediate consequences of our main results Theorem 2.1 and Theorem 2.3 where $M(x, y)=d(x, y)$.

Corollary 3.1 (see Karapınar and Samet [7]) Let $(X, d)$ be a complete metric space and $\alpha: X \times X \rightarrow[0,+\infty)$ be a transitive mapping. Suppose that $T: X \rightarrow X$ is a generalized $\alpha-\psi$-contractive mapping and satisfies the following conditions:

(i) $T$ is $\alpha$-admissible;

(ii) there exists $x_{0} \in X$ such that $\alpha\left(x_{0}, T x_{0}\right) \geq 1$;

(iii) $T$ is continuous.

Then $T$ has a fixed point, that is, there exists $z \in X$ such that $T z=z$.

Proof It is sufficient to take $\varphi(t)=1$ for all $t \geq 0$ in Theorem 2.3.

If one replaces $\varphi(t)=1$ for all $t \geq 0$ in Theorem 1.1, the following fixed point theorem is observed.

Corollary 3.2 (see Samet et al. [1]) Let $(X, d)$ be a complete metric space and $T: X \rightarrow X$ be an $\alpha-\psi$-contractive mapping satisfying the following conditions: 
(i) $T$ is $\alpha$-admissible;

(ii) there exists $x_{0} \in X$ such that $\alpha\left(x_{0}, T x_{0}\right) \geq 1$;

(iii) $T$ is continuous.

Then $T$ has a fixed point, that is, there exists $x^{*} \in X$ such that $T x^{*}=x^{*}$.

If we take $\alpha(x, y)=1$ for all $x, y \in X$ and $\psi(t)=k t$ for $k \in[0,1)$ in Theorem 1.1 , we derive the following result.

Corollary 3.3 (see Branciari [10]) Let $(X, d)$ be a complete metric space, $k \in[0,1)$, and let $T: X \rightarrow X$ be a mapping such that for each $x, y \in X$,

$$
\int_{0}^{d(T x, T y)} \varphi(t) d t \leq k \int_{0}^{d(x, y)} \varphi(t) d t
$$

where $\varphi \in \Phi$. Then $T$ has a unique fixed point $a \in X$ such that for each $x \in X, \lim _{n \rightarrow+\infty} T^{n} \times$ $x=a$.

The following corollary is concluded from Corollary 3.1 by taking $\alpha(x, y)=1$ for all $x, y \in X$.

Corollary 3.4 (see Karapinar and Samet [7]) Let $(X, d)$ be a complete metric space and $T: X \rightarrow X$ be a given mapping. Suppose that there exists a function $\psi \in \Psi$ such that

$$
d(T x, T y) \leq \psi(M(x, y))
$$

for all $x, y \in X$. Then $T$ has a unique fixed point.

By taking $\psi(t)=\lambda t$ for $\lambda \in[0,1)$ in Corollary 3.4, we get the next result.

Corollary 3.5 (see Ćirić [15]) Let $(X, d)$ be a complete metric space and $T: X \rightarrow X$ be a given mapping. Suppose that there exists a constant $\lambda \in(0,1)$ such that

$$
d(T x, T y) \leq \lambda \max \left\{d(x, y), \frac{d(x, T x)+d(y, T y)}{2}, \frac{d(x, T y)+d(y, T x)}{2}\right\}
$$

for all $x, y \in X$. Then $T$ has a unique fixed point.

Corollary 3.6 (see Hardy and Rogers [16]) Let $(X, d)$ be a complete metric space and $T$ : $X \rightarrow X$ be a given mapping. Suppose that there exist constants $A, B, C \geq 0$ with $(A+2 B+$ $2 C) \in(0,1)$ such that

$$
d(T x, T y) \leq A d(x, y)+B[d(x, T x)+d(y, T y)]+C[d(x, T y)+d(y, T x)]
$$

for all $x, y \in X$. Then $T$ has a unique fixed point.

For the proof of the above corollary, it is sufficient to chose $\lambda=\max \{A, B, C\}$ in Corollary 3.5.

The next two results are obvious consequences of Corollary 3.5. 
Corollary 3.7 (see Kannan [17]) Let $(X, d)$ be a complete metric space and $T: X \rightarrow X$ be a given mapping. Suppose that there exists a constant $\lambda \in(0,1 / 2)$ such that

$$
d(T x, T y) \leq \lambda[d(x, T x)+d(y, T y)]
$$

for all $x, y \in X$. Then $T$ has a unique fixed point.

Corollary 3.8 (see Chatterjea [18]) Let $(X, d)$ be a complete metric space and $T: X \rightarrow X$ be a given mapping. Suppose that there exists a constant $\lambda \in(0,1 / 2)$ such that

$$
d(T x, T y) \leq \lambda[d(x, T y)+d(y, T x)]
$$

for all $x, y \in X$. Then $T$ has a unique fixed point.

By taking $y=T x$ in Corollary 3.3, we obtain the following corollary.

Corollary 3.9 (Rhoades and Abbas [19]) Let T be a self-map of a complete metric space $(X, d)$ satisfying

$$
\int_{0}^{d\left(T x, T^{2} x\right)} \varphi(t) d t \leq k \int_{0}^{d(x, T x)} \varphi(t) d t
$$

for all $x \in X$ and $k \in[0,1)$, where $\varphi \in \Phi$. Then $T$ has a unique fixed point $a \in X$.

Corollary 3.10 (Berinde [20]) Let $(X, d)$ be a complete metric space and $T: X \rightarrow X$ be a given mapping. Suppose that there exists a function $\psi \in \Psi$ such that

$$
d(T x, T y) \leq \psi(d(x, y))
$$

for all $x, y \in X$. Then $T$ has a unique fixed point.

Proof Let $\alpha(x, y)=1$ for all $x, y \in X$ and $\varphi(t)=1$ for all $t \geq 0$ in Theorem 1.1. Then all the conditions of Theorem 1.1 are satisfied and the proof is completed.

It is evident that we have the celebrated result of Banach.

Corollary 3.11 (Banach [2]) Let $(X, d)$ be a complete metric space and $T: X \rightarrow X$ be a given mapping satisfying

$$
d(T x, T y) \leq k d(x, y) \quad \text { for all } x, y \in X
$$

where $k \in[0,1)$. Then $T$ has a unique fixed point.

\subsection{Fixed point theorems on ordered metric spaces}

Recently, there have been so many interesting developments in the field of existence of a fixed point in partially ordered sets. This idea was initiated by Ran and Reurings [21] where they extended the Banach contraction principle in partially ordered sets with some 
application to a matrix equation. Later, many remarkable results have been obtained in this direction (see, for example, [22-29] and the references cited therein). In this section, we will establish various fixed point results on a metric space endowed with a partial order. For this, we require the following concepts.

Definition 3.1 Let $(X, \preceq)$ be a partially ordered set and $T: X \rightarrow X$ be a given mapping. We say that $T$ is nondecreasing with respect to $\preceq$ if

$$
x, y \in X, \quad x \preceq y \quad \Rightarrow \quad T x \preceq T y \text {. }
$$

Definition 3.2 Let $(X, \preceq)$ be a partially ordered set. A sequence $\left\{x_{n}\right\} \subset X$ is said to be nondecreasing with respect to $\preceq$ if $x_{n} \preceq x_{n+1}$ for all $n$.

Definition 3.3 [7] Let $(X, \preceq)$ be a partially ordered set and $d$ be a metric on $X$. We say that $(X, \preceq, d)$ is regular if for every nondecreasing sequence $\left\{x_{n}\right\} \subset X$ such that $x_{n} \rightarrow x \in X$ as $n \rightarrow \infty$, there exists a subsequence $\left\{x_{n(k)}\right\}$ of $\left\{x_{n}\right\}$ such that $x_{n(k)} \preceq x$ for all $k$.

Now, we have the following result.

Corollary 3.12 Let $(X, \preceq)$ be a partially ordered set and $d$ be a metric on $X$ such that $(X, d)$ is complete. Let $T: X \rightarrow X$ be a nondecreasing mapping with respect to $\preceq$. Suppose that there exist functions $\psi \in \Psi$ and $\varphi \in \Phi$ such that for all $x, y \in X$ with $x \preceq y$, we have

$$
\int_{0}^{d(T x, T y)} \varphi(t) d t \leq \psi\left(\int_{0}^{M(x, y)} \varphi(t) d t\right)
$$

where $M(x, y)=\max \left\{d(x, y),\left[\frac{d(x, T x)+d(y, T y)}{2}\right],\left[\frac{d(x, T y)+d(y, T x)}{2}\right]\right\}$. Suppose also that the following conditions hold:

(i) there exists $x_{0} \in X$ such that $x_{0} \preceq T x_{0}$;

(ii) $T$ is continuous or $(X, \preceq, d)$ is regular.

Then $T$ has a fixed point. Moreover, if for all $x, y \in X$ there exists $z \in X$ such that $x \preceq z$ and $y \preceq z$, we have uniqueness of the fixed point.

Proof Consider the mapping $\alpha: X \times X \rightarrow[0, \infty)$ by

$$
\alpha(x, y)= \begin{cases}1 & \text { if } x \preceq y \\ 0 & \text { otherwise }\end{cases}
$$

Clearly, $\alpha$ is transitive. In view of the definition of $\alpha$, we infer that $T$ is an $\alpha-\psi$-contractive mapping of integral type, that is,

$$
\alpha(x, y) \int_{0}^{d(T x, T y)} \varphi(t) d t \leq \psi\left(\int_{0}^{M(x, y)} \varphi(t) d t\right)
$$

for all $x, y \in X$. From condition (i), we have $\alpha\left(x_{0}, T x_{0}\right) \geq 1$. Now, we proceed to show that $T$ is $\alpha$-admissible. For this, let $\alpha(x, y) \geq 1$ for all $x, y \in X$. Moreover, owing to the monotone property of $T$, we have, for all $x, y \in X$,

$$
\alpha(x, y) \geq 1 \quad \Rightarrow \quad x \preceq y \quad \Rightarrow \quad T x \preceq T y \quad \Rightarrow \quad \alpha(T x, T y) \geq 1 .
$$


Thus, $T$ is $\alpha$-admissible. Now, if $T$ is continuous, we obtain the existence of a fixed point from Theorem 2.3. Now, assume that $(X, \preceq, d)$ is regular. Suppose that $\left\{x_{n}\right\}$ is a sequence in $X$ such that $\alpha\left(x_{n}, x_{n+1}\right) \geq 1$ for all $n$ and $x_{n} \rightarrow x \in X$ as $n \rightarrow \infty$. Due to the fact that the space $(X, \preceq, d)$ is regular, there exists a subsequence $\left\{x_{n(k)}\right\}$ of $\left\{x_{n}\right\}$ such that $x_{n(k)} \preceq x$ for all $k$. Owing to the definition of $\alpha$, we get that $\alpha\left(x_{n(k)}, x\right) \geq 1$ for all $k$. In this case, we get the existence of a fixed point from Theorem 2.4. Now, we have to show the uniqueness of the fixed point. For this, let $x, y \in X$. By hypothesis, there exists $z \in X$ such that $x \preceq z$ and $y \preceq z$, which implies from the definition of $\alpha$ that $\alpha(x, z) \geq 1$ and $\alpha(y, z) \geq 1$. Therefore, we obtain the uniqueness of the fixed point from Theorem 2.6.

We can now easily derive the following results from Corollary 3.12.

Corollary 3.13 (Shahi et al. [9]) Let $(X, \preceq)$ be a partially ordered set and d be a metric on $X$ such that $(X, d)$ is complete. Let $T: X \rightarrow X$ be a nondecreasing mapping with respect to $\preceq$. Suppose that there exists a function $\psi \in \Psi$ such that for all $x, y \in X$ with $x \preceq y$, we have

$$
\int_{0}^{d(T x, T y)} \varphi(t) d t \leq \psi\left(\int_{0}^{d(x, y)} \varphi(t) d t\right)
$$

where $\varphi \in \Phi$. Suppose also that the following conditions hold:

(i) there exists $x_{0} \in X$ such that $x_{0} \preceq T x_{0}$;

(ii) $T$ is continuous or $(X, \preceq, d)$ is regular.

Then $T$ has a fixed point. Moreover, if for all $x, y \in X$ there exists $z \in X$ such that $x \preceq z$ and $y \preceq z$, we have uniqueness of the fixed point.

Corollary 3.14 (Karapınar and Samet [7]) Let $(X, \preceq)$ be a partially ordered set and d be a metric on $X$ such that $(X, d)$ is complete. Let $T: X \rightarrow X$ be a nondecreasing mapping with respect to $\preceq$. Suppose that there exists a function $\psi \in \Psi$ such that

$$
d(T x, T y) \leq \psi(M(x, y))
$$

for all $x, y \in X$ with $x \preceq y$. Suppose also that the following conditions hold:

(i) there exists $x_{0} \in X$ such that $x_{0} \preceq T x_{0}$;

(ii) $T$ is continuous or $(X, \preceq, d)$ is regular.

Then $T$ has a fixed point. Moreover, iffor all $x, y \in X$ there exists $z \in X$ such that $x \preceq z$ and $y \preceq z$, we have uniqueness of the fixed point.

Proof By taking $\varphi(t)=1$ for all $t \geq 0$ in Corollary 3.12, we get the proof of this corollary.

Corollary 3.15 (Karapınar and Samet [7]) Let $(X, \preceq)$ be a partially ordered set and d be a metric on $X$ such that $(X, d)$ is complete. Let $T: X \rightarrow X$ be a nondecreasing mapping with respect to $\preceq$. Suppose that there exists a function $\psi \in \Psi$ such that

$$
d(T x, T y) \leq \psi(d(x, y))
$$

for all $x, y \in X$ with $x \preceq y$. Suppose also that the following conditions hold: 
(i) there exists $x_{0} \in X$ such that $x_{0} \preceq T x_{0}$;

(ii) $T$ is continuous or $(X, \preceq, d)$ is regular.

Then $T$ has a fixed point. Moreover, if for all $x, y \in X$ there exists $z \in X$ such that $x \preceq z$ and $y \preceq z$, we have uniqueness of the fixed point.

Proof By taking $\varphi(t)=1$ for all $t \geq 0$ in Corollary 3.13, we get the proof of this corollary.

Corollary 3.16 (Shahi et al. [9]) Let $(X, \preceq)$ be a partially ordered set and $d$ be a metric on $X$ such that $(X, d)$ is complete. Let $T: X \rightarrow X$ be a nondecreasing mapping with respect to $\preceq$. Suppose that there exists a function $\psi \in \Psi$ such that for all $x, y \in X$ with $x \preceq y$, we have

$$
\int_{0}^{d(T x, T y)} \varphi(t) d t \leq k \int_{0}^{d(x, y)} \varphi(t) d t
$$

where $\varphi \in \Phi$. Suppose also that the following conditions hold:

(i) there exists $x_{0} \in X$ such that $x_{0} \preceq T x_{0}$;

(ii) $T$ is continuous or $(X, \preceq, d)$ is regular.

Then $T$ has a fixed point. Moreover, if for all $x, y \in X$ there exists $z \in X$ such that $x \preceq z$ and $y \preceq z$, we have uniqueness of the fixed point.

Proof By taking $\psi(t)=k t$ for all $t \geq 0$ and some $k \in[0,1)$ in Corollary 3.13, we get the proof of this corollary.

Corollary 3.17 (Ran and Reurings [21], Nieto and Rodriguez-Lopez [29]) Let (X, $)$ be a partially ordered set and $d$ be a metric on $X$ such that $(X, d)$ is complete. Let $T: X \rightarrow X$ be a nondecreasing mapping with respect to $\preceq$. Suppose that there exists a constant $k \in(0,1)$ such that

$$
d(T x, T y) \leq k d(x, y)
$$

for all $x, y \in X$ with $x \preceq y$. Suppose also that the following conditions hold:

(i) there exists $x_{0} \in X$ such that $x_{0} \preceq T x_{0}$;

(ii) $T$ is continuous or $(X, \preceq, d)$ is regular.

Then $T$ has a fixed point. Moreover, iffor all $x, y \in X$ there exists $z \in X$ such that $x \preceq z$ and $y \preceq z$, we have uniqueness of the fixed point.

Proof Taking $\varphi(t)=1$ for all $t \geq 0$ in Corollary 3.16, we get the proof of this corollary.

Corollary 3.18 (see Karapınar and Samet [7]) Let $(X, \preceq)$ be a partially ordered set and d be a metric on $X$ such that $(X, d)$ is complete. Let $T: X \rightarrow X$ be a nondecreasing mapping with respect to $\preceq$. Suppose that there exists a constant $\lambda \in(0,1)$ such that

$$
d(T x, T y) \leq \lambda \max \left\{d(x, y), \frac{d(x, T x)+d(y, T y)}{2}, \frac{d(x, T y)+d(y, T x)}{2}\right\}
$$

for all $x, y \in X$ with $x \preceq y$. Suppose also that the following conditions hold: 
(i) there exists $x_{0} \in X$ such that $x_{0} \preceq T x_{0}$;

(ii) $T$ is continuous or $(X, \preceq, d)$ is regular.

Then $T$ has a fixed point. Moreover, if for all $x, y \in X$ there exists $z \in X$ such that $x \preceq z$ and $y \preceq z$, we have uniqueness of the fixed point.

Corollary 3.19 (see Karapınar and Samet [7]) Let $(X, \preceq)$ be a partially ordered set and $d$ be a metric on $X$ such that $(X, d)$ is complete. Let $T: X \rightarrow X$ be a nondecreasing mapping with respect to $\preceq$. Suppose that there exist constants $A, B, C \geq 0$ with $(A+2 B+2 C) \in(0,1)$ such that

$$
d(T x, T y) \leq A d(x, y)+B[d(x, T x)+d(y, T y)]+C[d(x, T y)+d(y, T x)]
$$

for all $x, y \in X$ with $x \preceq y$. Suppose also that the following conditions hold:

(i) there exists $x_{0} \in X$ such that $x_{0} \preceq T x_{0}$;

(ii) $T$ is continuous or $(X, \preceq, d)$ is regular.

Then $T$ has a fixed point. Moreover, if for all $x, y \in X$ there exists $z \in X$ such that $x \preceq z$ and $y \preceq z$, we have uniqueness of the fixed point.

Corollary 3.20 (see Karapınar and Samet [7]) Let $(X, \preceq)$ be a partially ordered set and $d$ be a metric on $X$ such that $(X, d)$ is complete. Let $T: X \rightarrow X$ be a nondecreasing mapping with respect to $\preceq$. Suppose that there exists a constant $\lambda \in(0,1 / 2)$ such that

$$
d(T x, T y) \leq \lambda[d(x, T x)+d(y, T y)]
$$

for all $x, y \in X$ with $x \preceq y$. Suppose also that the following conditions hold:

(i) there exists $x_{0} \in X$ such that $x_{0} \preceq T x_{0}$;

(ii) $T$ is continuous or $(X, \preceq, d)$ is regular.

Then $T$ has a fixed point. Moreover, if for all $x, y \in X$ there exists $z \in X$ such that $x \preceq z$ and $y \preceq z$, we have uniqueness of the fixed point.

Corollary 3.21 (see Karapinar and Samet [7]) Let $(X, \preceq)$ be a partially ordered set and d be a metric on $X$ such that $(X, d)$ is complete. Let $T: X \rightarrow X$ be a nondecreasing mapping with respect to $\preceq$. Suppose that there exists a constant $\lambda \in(0,1 / 2)$ such that

$$
d(T x, T y) \leq \lambda[d(x, T y)+d(y, T x)]
$$

for all $x, y \in X$ with $x \preceq y$. Suppose also that the following conditions hold:

(i) there exists $x_{0} \in X$ such that $x_{0} \preceq T x_{0}$;

(ii) $T$ is continuous or $(X, \preceq, d)$ is regular.

Then $T$ has a fixed point. Moreover, iffor all $x, y \in X$ there exists $z \in X$ such that $x \preceq z$ and $y \preceq z$, we have uniqueness of the fixed point. 


\section{Author details}

${ }^{1}$ Department of Mathematics, Atilim University, Incek, Ankara, 06836, Turkey. ${ }^{2}$ Nonlinear Analysis and Applied Mathematics Research Group (NAAM), King Abdulaziz University, Jeddah, Saudi Arabia. ${ }^{3}$ School of Mathematics and Computer Applications, Thapar University, Patiala, Punjab 147004, India. ${ }^{4}$ Department Mathematics and Computer Science, Cankaya University, Ankara, Turkey.

\section{Acknowledgements}

The authors are grateful to the reviewers for their careful reviews and useful comments. The first author was supported by the Research Center, College of Science, King Saud University.

\section{Received: 7 February 2014 Accepted: 16 April 2014 Published: 06 May 2014}

\section{References}

1. Samet, B, Vetro, C, Vetro, P: Fixed point theorem for $\alpha-\psi$-contractive type mappings. Nonlinear Anal. 75, 2154-2165 (2012)

2. Banach, S: Sur les operations dans les ensembles abstraits et leur application aux equations integrales. Fundam Math. 3, 133-181 (1922)

3. Ali, MU, Kamran, T: On $\left(\alpha^{*}, \psi\right)$-contractive multi-valued mappings. Fixed Point Theory Appl. 2013, 137 (2013). doi:10.1186/1687-1812-2013-137

4. Berzig, M, Rus, M: Fixed point theorems for $\alpha$-contractive mappings of Meir-Keeler type and applications. arXiv:1303.5798 [math.GN]

5. Jleli, M, Karapınar, E, Samet, B: Best proximity points for generalized $\alpha$ - $\psi$-proximal contractive type mappings. J. Appl. Math. 2013, Article ID 534127 (2013)

6. Jleli, M, Karapınar, E, Samet, B: Fixed point results for $\alpha$ - $\psi_{\lambda}$-contractions on gauge spaces and applications. Abstr Appl. Anal. 2013, Article ID 730825 (2013)

7. Karapınar, E, Samet, B: Generalized $\alpha-\psi$-contractive type mappings and related fixed point theorems with applications. Abstr. Appl. Anal. 2012, Article ID 793486 (2012). doi:10.1155/2012/793486

8. Mohammadi, B, Rezapour, S, Shahzad, N: Some results on fixed points of $\alpha$ - $\psi$-Ciric generalized multifunctions. Fixed Point Theory Appl. 2013, 24 (2013). doi:10.1186/1687-1812-2013-24

9. Shahi, P, Kaur, J, Bhatia, SS: Fixed point theorems for $\alpha-\psi$-contractive type mappings of integral type with applications. J. Nonlinear Convex Anal. (accepted)

10. Branciari, A: A fixed point theorem for mappings satisfying a general contractive condition of integral type. Int. J. Math. Math. Sci. 29(9), 531-536 (2002)

11. Rus, IA: Generalized Contractions and Applications. Cluj University Press, Cluj-Napoca (2001)

12. Bianchini, RM, Grandolfi, M: Transformazioni di tipo contracttivo generalizzato in uno spazio metrico. Atti Accad. Naz. Lincei, Rend. Cl. Sci. Fis. Mat. Nat. (7) 45, 212-216 (1968)

13. Proinov, PD: A generalization of the Banach contraction principle with high order of convergence of successive approximations. Nonlinear Anal. TMA 67, 2361-2369 (2007)

14. Proinov, PD: New general convergence theory for iterative processes and its applications to Newton Kantorovich type theorems. J. Complex. 26, 3-42 (2010)

15. Ćirić, LB: Fixed points for generalized multi-valued contractions. Mat. Vesn. 9(24), 265-272 (1972)

16. Hardy, GE, Rogers, TD: A generalization of a fixed point theorem of Reich. Can. Math. Bull. 16, $201-206$ (1973)

17. Kannan, R: Some results on fixed points. Bull. Calcutta Math. Soc. 10, 71-76 (1968)

18. Chatterjea, SK: Fixed-point theorems. C. R. Acad. Bulgare Sci. 25, 727-730 (1972)

19. Rhoades, BE, Abbas, M: Maps satisfying generalized contractive conditions of integral type for which $F(T)=F\left(T^{n}\right)$. Int. J. Pure Appl. Math. 45(2), 225-231 (2008)

20. Berinde, V: Iterative Approximation of Fixed Points. Editura Efemeride, Baia Mare (2002)

21. Ran, ACM, Reurings, MCB: A fixed point theorem in partially ordered sets and some applications to matrix equations. Proc. Am. Math. Soc. 132, 1435-1443 (2004)

22. Agarwal, RP, El-Gebeily, MA, O'Regan, D: Generalized contractions in partially ordered metric spaces. Appl. Anal. 87, $1-8(2008)$

23. Berinde, $V$, Borcut, M: Tripled fixed point theorems for contractive type mappings in partially ordered metric spaces. Nonlinear Anal. 74, 4889-4897 (2011)

24. Berinde, V: Coupled fixed point theorems for generalized symmetric Meir-Keeler contractions in ordered metric spaces. arXiv:1103.5289 [math.FA]

25. Bhaskar, TG, Lakshmikantham, V: Fixed point theory in partially ordered metric spaces and applications. Nonlinear Anal. 65, 1379-1393 (2006)

26. Lakshmikantham, V, Ćirić, L: Coupled fixed point theorems for nonlinear contractions in partially ordered metric spaces. Nonlinear Anal. 70, 4341-4349 (2009)

27. Luong, NV, Thuan, NX: Coupled fixed points in partially ordered metric spaces and application. Nonlinear Anal, Theory Methods Appl. 74, 983-992 (2011)

28. Nashine, HK, Samet, B: Fixed point results for mappings satisfying $(\psi, \varphi)$-weakly contractive condition in partially ordered metric spaces. Nonlinear Anal., Theory Methods Appl. 74, 2201-2209 (2011)

29. Nieto, JJ, Rodriguez-Lopez, R: Contractive mapping theorems in partially ordered sets and applications to ordinary differential equations. Order 22, 223-239 (2005)

\subsection{6/1029-242X-2014-160}

Cite this article as: Karapınar et al.: Generalized $\alpha-\psi$-contractive type mappings of integral type and related fixed point theorems. Journal of Inequalities and Applications 2014, 2014:160 